\title{
Relevés de fluctuations sur quelques glaciers des Alpes Françaises
}

\author{
Fluctuation surveying on some glaciers in the French Alps
}

\author{
par Louis Reynaud et Christian Vincent, \\ Laboratoire de Glaciologie et Géophysique de l'Environnement, CNRS, Grenoble.
}

Glacial fluctuation measurements by the Laboratoire de Glaciologie du CNRS (LGGE) are yearly made on some ten glaciers of the French Alps. The aim is to obtain a data base on mass balances and glacial dynamics parameters (lengths, altitudes and velocities). Thanks to ancient measurements of the Eaux et Forêts and to modern surveys, we have at our disposal some series to test physical models of the glacier variations.

\section{I $\square$ INTRODUCTION}

Le programme actuel d'observations systématiques des fluctuations glaciaires du Laboratoire de Glaciologie (LGGE-CNRS) dans les Alpes Françaises comprend un échantillonnage d'une dizaine de glaciers répartis dans les principaux massifs du Mont-Blanc, de la Vanoise, des Grandes Rousses et des Ecrins. Ces glaciers ont été choisis dans ces différents massifs, soit pour leurs caractéristiques liées à leur dynamique vigoureuse (Bandes de Forbes, ondes cinématiques), soit pour leur disposition plus adaptée à la détermination du bilan de masse et de ses fluctuations annuelles et saisonnières. Dans ce choix, l'accessibilité en hiver ou été tient une bonne part, de même que l'existence de mesures antérieures. En effet les relevés anciens, principalement ceux des Eaux et Forêts (E\&F), permettent de bénéficier d'un long héritage de connaissances de variations (longueurs, altitudes et vitesses d'écoulement) et de les valoriser fortement en y adjoignant des relevés modernes de bilan de masse, sur de larges gammes d'altitudes, de façon à disposer d'une description la plus complète possible.

Le but poursuivi vise à une meilleure documentation des réactions des glaciers comme celles annuelles de leurs bilans de masse qui constituent des mesures directes du changement climatique et de sa répartition spatiale. En outre, ces modifications de bilan constituent le signal d'entrée des variations glaciaires qui se traduisent par des variations de vitesses, d'altitudes et d'extension des glaciers. Ces données de base sont indispensables à la compréhension des mécanismes fondamentaux, à leur modélisation physique réaliste et au test des modélisations sur une authentique vérité terrain.

Dans cet exposé, nous ne présenterons qu'une partie des résultats obtenus dans chaque massif, en nous attachant plus particulièrement à quelques longues séries de relevés.

\section{MASSIF DU MONT BLANC}

\subsection{Le Glacier d'Argentière}

Le suivi des fluctuations du glacier d'Argentière a débuté en 1903 dans le dispositif classique adopté à cette époque par les Eaux et Forêts (E \& F) [1], qui visait à étudier l'état de santé d'un secteur de la langue terminale délimité par deux profils transversaux fixes. Les relevés consistaient à déterminer par topographie les positions successives des repères (pierres peintes) sur ces deux profils pour en déduire les altitudes et les vitesses annuelles. Probablement que cette méthode visait à établir le bilan du secteur par la conservation de la masse, à une époque où l'on ne savait pas encore implanter des balises assez profondément pour mesurer les 8 à $12 \mathrm{~m} / \mathrm{an}$ d'ablation sur les langues terminales. Mais, pour appliquer la conservation de masse, il faut aussi disposer des épaisseurs, ce qui ne sera possible qu'avec les moyens géophysiques modernes comme la sismique où le radar, ainsi que du calcul des vitesses en profondeur [2].

Sur ce glacier où Électricité de France (EDF) désirait capter l'eau du torrent sous-glaciaire en altitude, ces mesures E\&F ont été heureusement reprises et étendues à trois profils situés sur le plateau supérieur dès 1960. A partir des années 1970, c'est la société franco-suisse Emosson SA qui a poursuivi les relevés en les confiant dès 1975 au Laboratoire de glaciologie du CNRS (LGGE).

L'ensemble des relevés effectués sur le glacier d'Argentière constitue une observation assez complète en incluant aujourd'hui :

- les mesures de bilan de masse hivernaux et estivaux (de $3400 \mathrm{~m}$ à $1500 \mathrm{~m}$ d'altitude) $[3,4]$,

- les mesures des vitesses aux 5 profils, ainsi que sur des lignes longitudinales pour décrire plus en détails la dyna- 


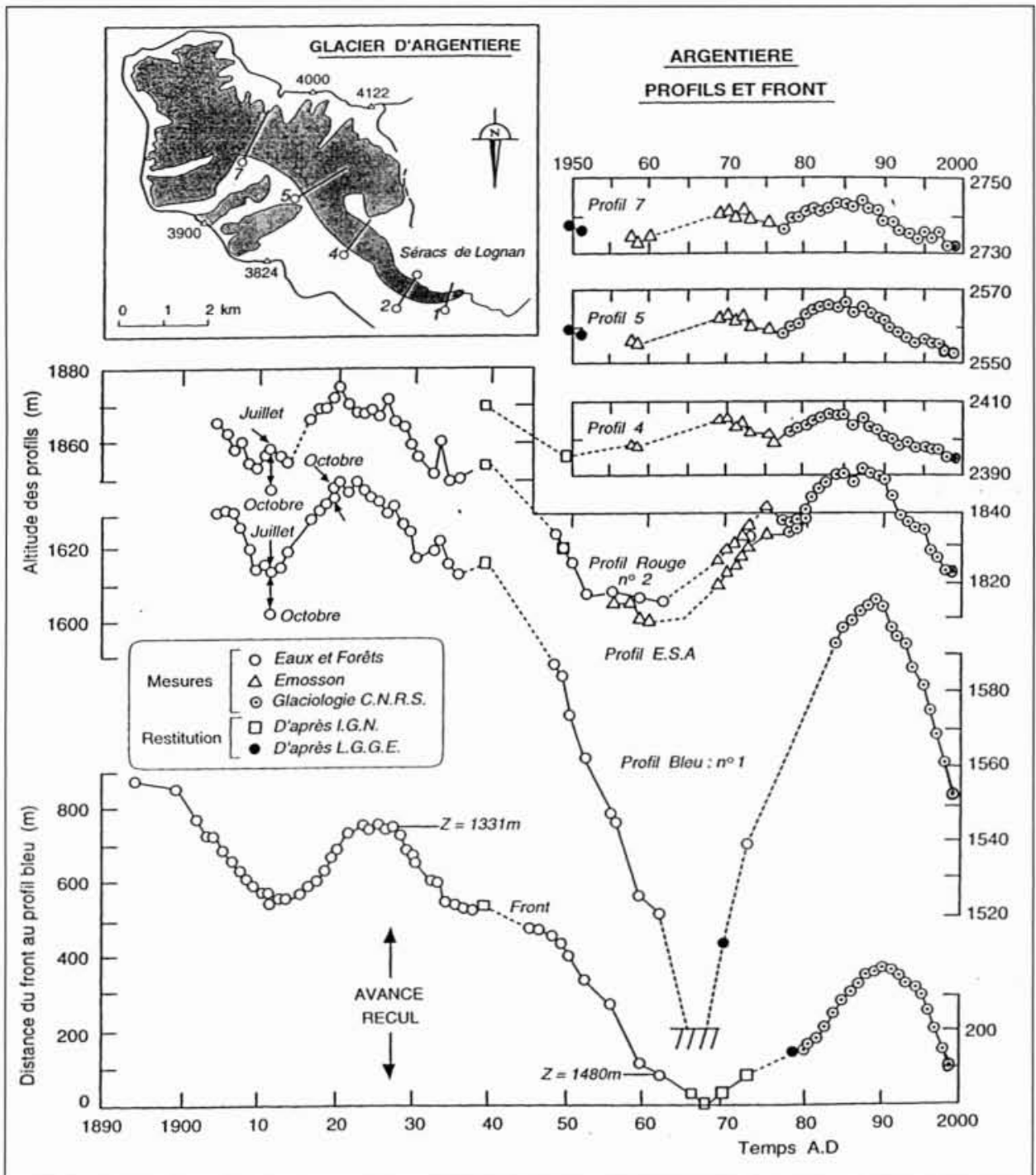

1. Glacier d'Argentière : Variations des Altitudes moyennes aux cinq profils transversaux et variations de longueur.

mique du glacier et ses modifications au cours du temps.

Sur le graphique $n^{\circ} 1$ ne sont présentées que les variations d'altitudes moyennes aux cinq profils transversaux en même temps que les variations de longueur.

Cet ensemble de relevés annuels d'altitudes moyennes montre principalement :

- une faible amplitude de variation sur les trois profils du plateau supérieur, depuis 1958 avec un fort synchronisme,

- en revanche, les deux profils inférieurs, à l'aval de la chute de glace (les profils E\&F) montrent des réactions plus amples qui se produisent avec un décalage dans le temps qui se poursuit jusqu'au front du glacier.

On remarque que ces faibles variations du plateau supérieur, en contraste avec celles de la partie basse du glacier, sont probablement une caractéristique des glaciers partagés en deux par une chute de séracs, car on retrouve ce comportement sur d'autres glaciers du même type comme le glacier Blanc ou celui de la Girose (Écrins).

\subsection{La Mer de Glace}

Sur la figure 2 sont portées les variations d'altitudes sur les $8 \mathrm{~km}$ de la langue inférieure de la Mer de Glace ainsi que les variations de longueur ; les chiffres portés sous chaque profil sont les abscisses curvilignes comptées à partir d'une origine choisie au centre de la chute de sérac du Géant.

Ces mesures ont été entreprises par Joseph Vallot en 1890 , [5], puis poursuivies à partir de 1912 par les E\&F en rajoutant des profils à l'amont, comme celui de Trélaporte et du Tacul, à mesure que le recul du front faisait disparaître les profils inférieurs du Chapeau et du Mauvais pas.

Même si cette série est incomplète entre 1900 et 1910 et limitée à la langue d'ablation, elle fournit néanmoins une série séculaire assez détaillée des variations d'altitude à la surface où Lliboutry et Reynaud [6,7] ont déjà reconnu des propagations d'ondes cinématiques.

C'est à notre connaissance le seul suivi glaciaire qui permette de faire ce type d'observation sur une aussi longue période.

Pour pouvoir en tirer des conclusions plus complètes, il faudrait disposer des autres paramètres de fluctuation, c'està-dire le bilan de masse ainsi que les vitesses.

Les bilans de masse, partiellement mesurés, peuvent être complétés avec les reconstitutions de leurs fluctuations. Pour les vitesses, les Bandes de Forbes fournissent une série reconstituée qui permet de remonter jusqu'aux années 1890 [8]. 


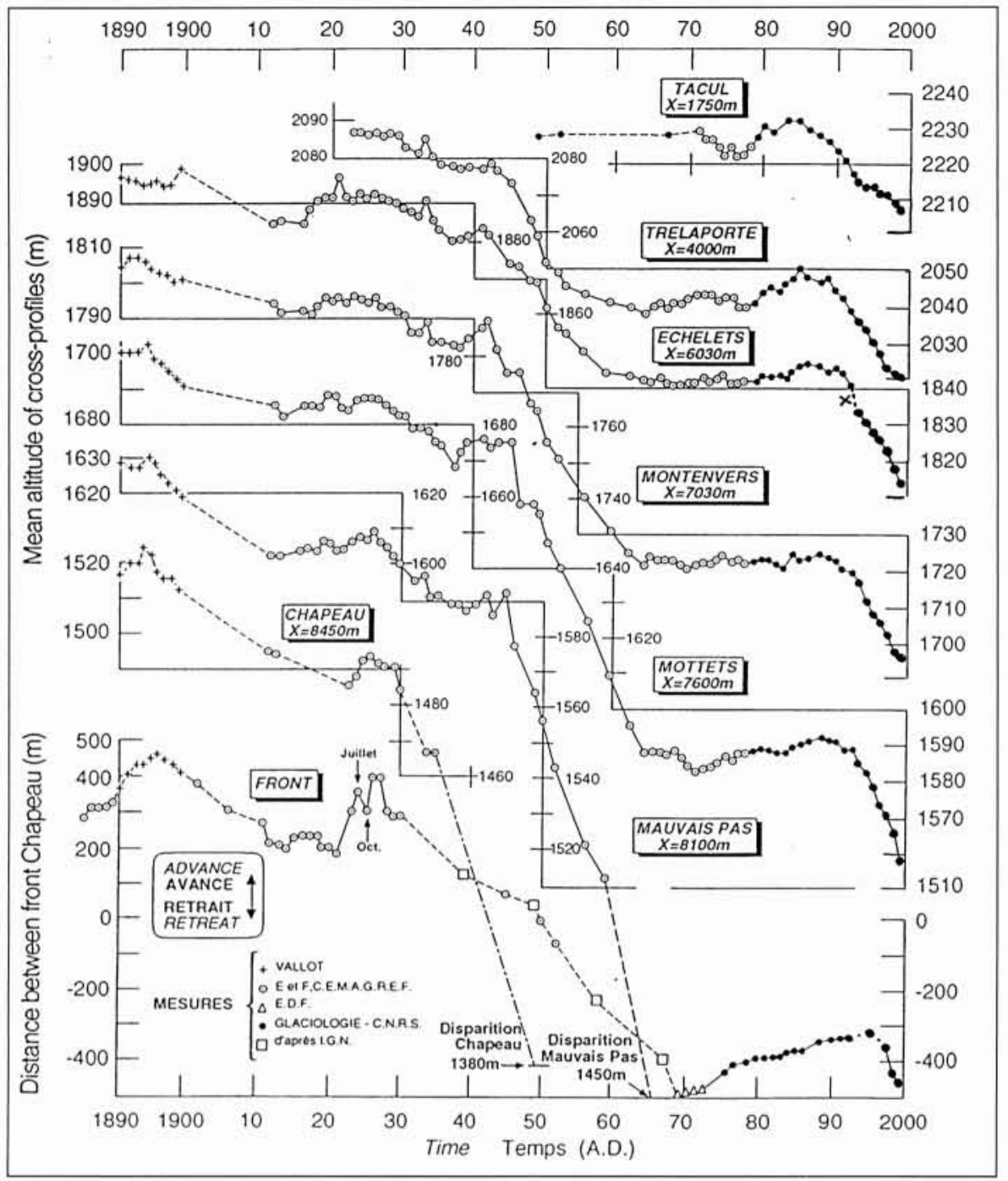

2. Mer de Glace : Altitudes moyennes aux cinq profils transversaux et variations de longueur.

\subsection{Variations de longueur dans le massif du Mont-Blanc}

Sur le même versant nord du Mont-Blanc nous disposons de relevés sur quatre glaciers voisins : Bossons, Argentière, Trient et Mer de Glace (cf. fig. 3). Ces mesures de longueur annuelles n'ont débuté, de façon continue que vers les années 1860-70. A part la série Suisse du Trient, remarquablement régulière, nous avons eu à compléter les séries françaises par quelques déterminations tirées de la photogrammétrie aérienne (IGN et Cemagref).

On peut noter que depuis 1997 le glacier des Bossons fait une petite réavancée d'une vingtaine de mètres par an, alors que la Mer de Glace a clairement débuté son retrait en laissant sur place une épaisse moraine frontale qui retient un petit lac à l'amont.

A première vue, ces quatre glaciers, de même type morphologique à chute de séracs, montrent de vigoureuses variations au cours du dernier siècle, soit lors des réavancées presque synchrones à première vue, comme celles de 1890 , 1920 et 1980, entrecoupées de reculs dont celui qui a débuté en 1940 fut très marqué.

Toutefois, en y regardant de plus près et en notant le début des réavancées, on obtient le graphique du bas, où il apparaît que le glacier des Bossons est le premier à réagir à chacune des 3 crues, suivi 4 à 7 ans plus tard par le tandem Argentière-Trient, tandis qu'il faut attendre 11 à 15 années pour que la Mer de Glace réagisse à son tour.

Ce comportement à peu près semblable lors des 3 crues de ce dernier siècle fournit une idée des temps de réponse des différents appareils qui se classent ici en raison de la taille depuis le plus court, celui des Bossons, jusqu'au plus long, celui de la Mer de Glace.

Parmi les grands glaciers du Mont-Blanc possédant une longue série de mesures, on peut compter celui de Tré la Tête, situé au sud du massif, dans la Réserve Naturelle des Contamines-Montjoie.

Sur la figure $n^{\circ} 4$ ont été rassemblées toutes les mesures de position du front depuis la première répertoriée dans la Mappe Sarde (cadastre) de 1730 . Entre 1900 et 1950 ce glacier a bénéficié du suivi des E\&F, avec suivi des vitesses et altitudes sur 5 profils transversaux [2].

Depuis 1950, les variations de longueurs ne sont plus effectuées directement et on ne dispose que de déterminations tirées de cartes ou photogrammétries aériennes. C'est pourquoi les Réserves Naturelles de Haute Savoie (APEGE, Annecy) ont décidé de poursuivre cette longue série en réinstallant en 1999 un système de repères topographiés dans 


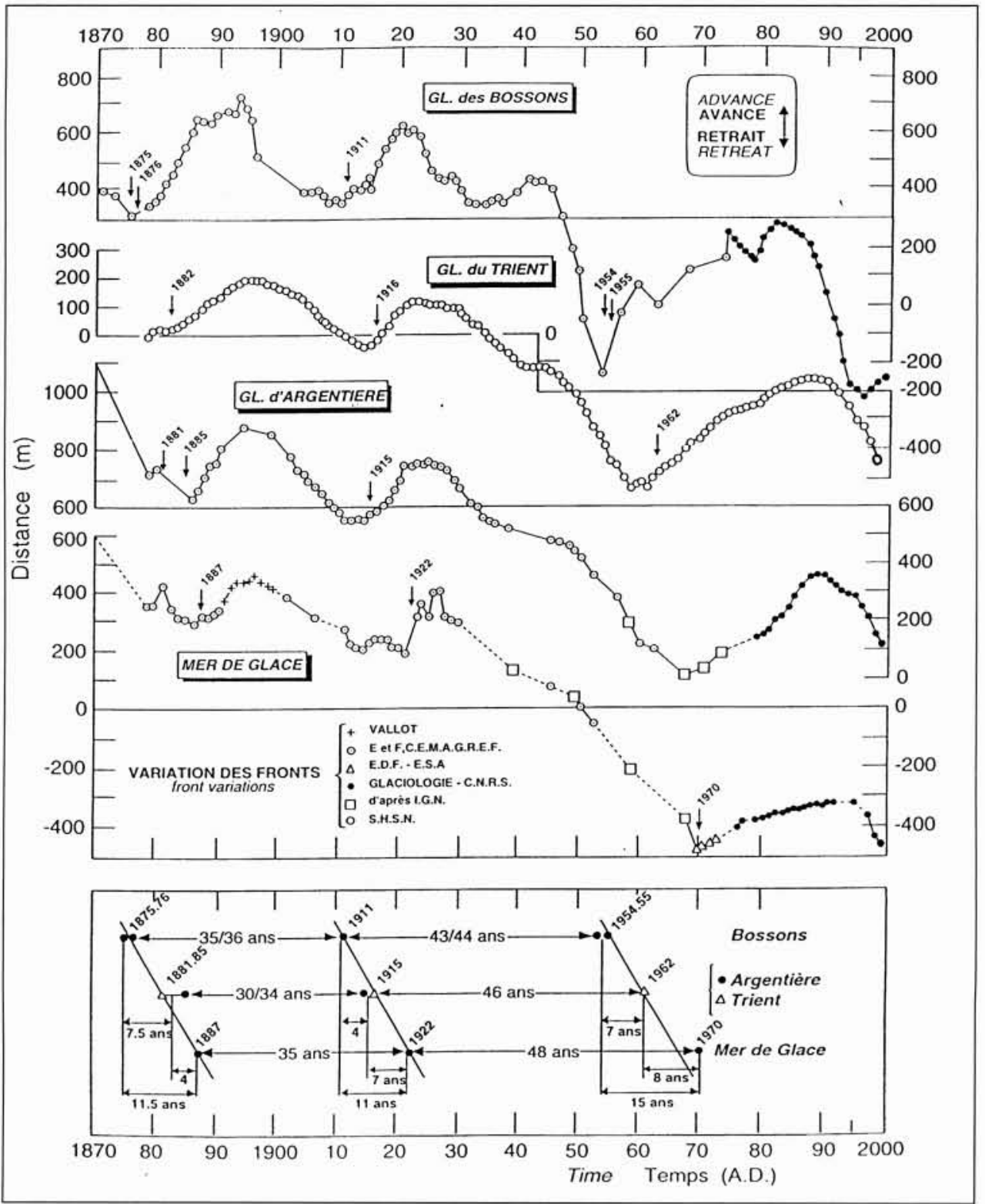

3. Variations de longueurs de 4 glaciers de la face nord du Mont-Blanc.

le système des $\mathrm{E} \& \mathrm{~F}$ et en relevant régulièrement les variations de longueur annuelles

\section{III — LE MASSIF DE LA VANOISE}

Dans ce massif, le glacier de Gébroulaz est le seul appareil suivi en détail. Il a été choisi comme représentatif de ce massif car il a bénéficié par le passé du suivi des E\&F depuis 1907 dans le système classique des relevés à deux profils transversaux et du levé du front [9]. Aujourd'hui, ce glacier, faisant partie du programme de suivi des bilans de masse, est équipé d'un réseau de relevés complet de bilans (hiver et été) (cf. Fig n5).

Sur les figures $n^{\circ} 6$ et 7 sont présentées respectivement les variations d'altitude et de vitesses pour toute la période 1907-1999.

Ces deux graphiques d'évolution au cours du temps révèlent clairement le comportement d'un glacier aux fluctuations du climat par ses modifications combinées d'altitude et de vitesse lors d'une crue, plus particulièrement dans la période 1910 à 1930 , où la documentation est plus complète.
Alors que le changement d'altitude de la surface reste modéré aux deux profils, on constate une accélération très importante des vitesses passant de $5 \mathrm{~m} / \mathrm{an}$ à plus de $30 \mathrm{~m} / \mathrm{an}$, cela en une dizaine d'années seulement.

Une fois la décrue amorcée, les vitesses retombent très rapidement à leur niveau primitif.

Pour la seconde crue du $\mathrm{XX}^{\circ}$ siècle, celle des années 1980 , il semble qu'il en ait été de même, bien qu'on ait manqué les relevés du début du phénomène.

Ce type de relevés est extrêmement intéressant car d'une part il est net qu'il n'est pas réservé au seul glacier de Gébroulaz : c'est un comportement dynamique que l'on retrouve sur tous les glaciers pour lesquels on dispose de relevés du type de ceux des E\&F.

D'autre part ce comportement n'est pas prévu par les modèles mécaniques avec lesquels on modélise généralement les fluctuations des glaciers [10]. En effet si ces modèles prennent bien en compte la déformation de la glace, ils ne savent pas rendre compte du glissement à la base qui est pourtant le processus prépondérant pour l'écoulement des glaciers tempérés. 


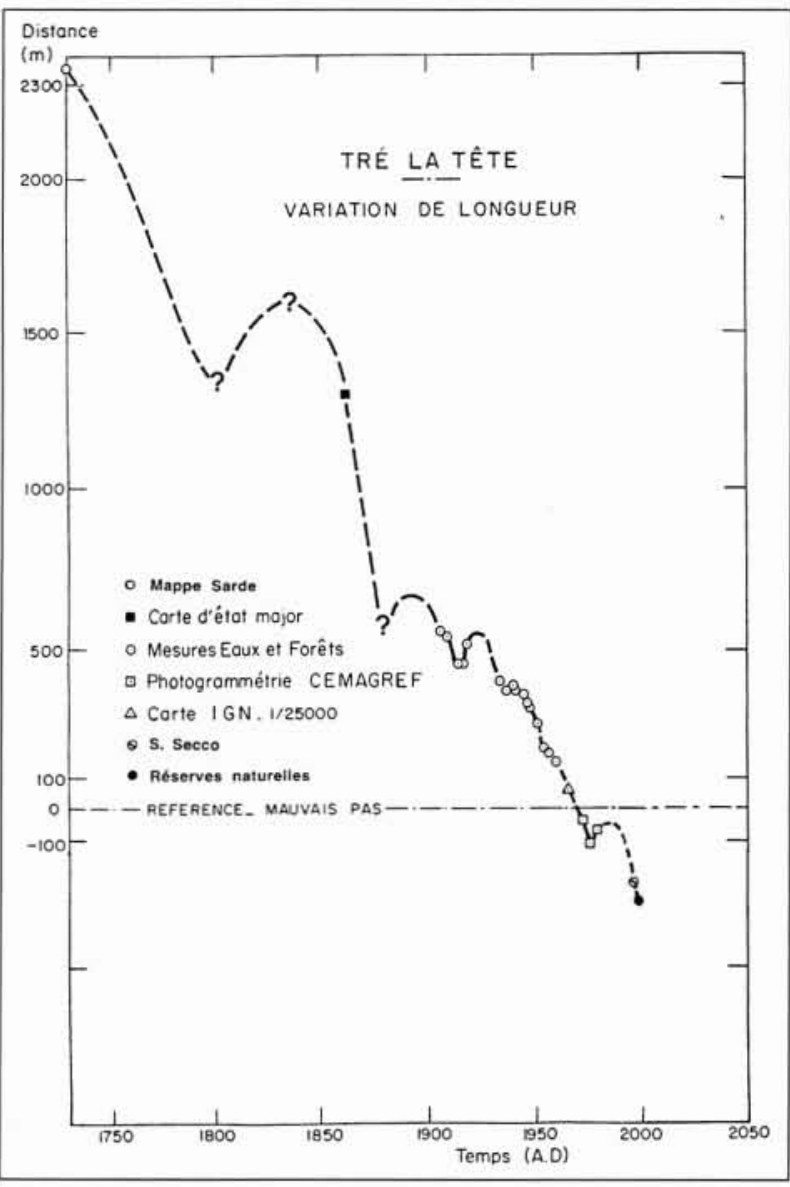

4. Variations de longueur du glacier de Tré la Tête : suivi repris en 1999 par les Réserves Naturelles de Haute Savoie (APEGE, Annecy).

On dispose donc là d'un ensemble de relevés indispensable à la compréhension des mécanismes réels mis en jeu dans la dynamique glaciaire et spécialement lors des crues glaciaires comme on en a eu seulement deux fois l'occasion au cours de ce dernier siècle : c'est pourquoi il conviendrait d'en assurer le suivi régulier sans manquer la prochaine crue.

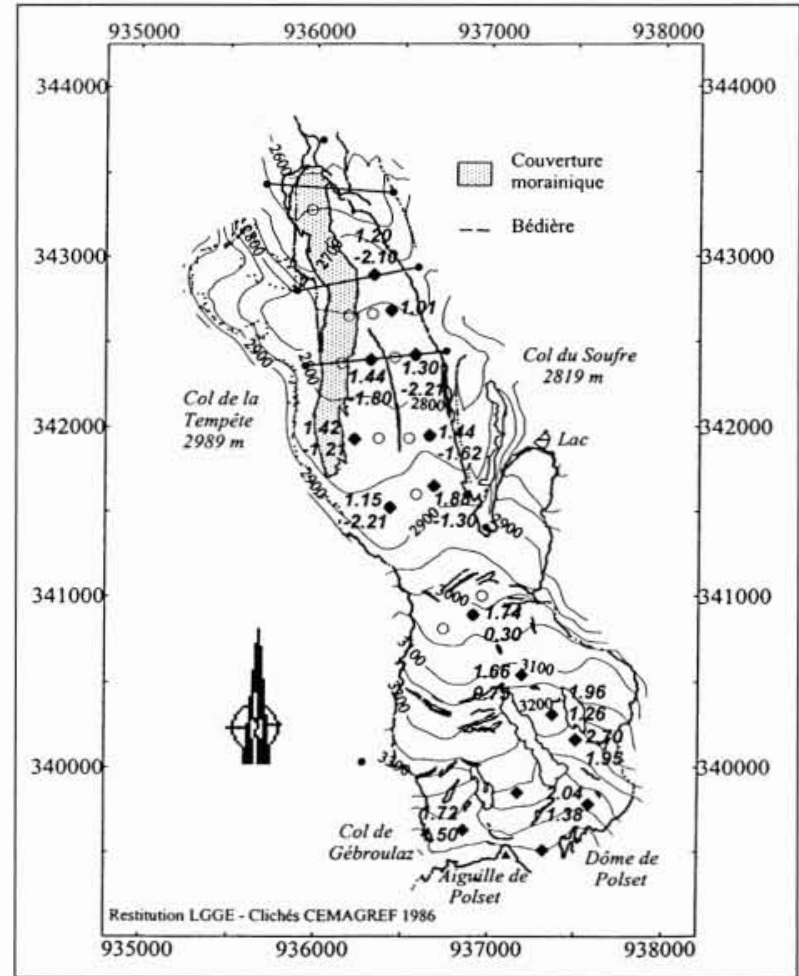

5. Carte de localisation sur le glacier de Gébroulaz des mesures de bilans en 1998-99.

Les chiffres portés près des losanges noirs, sont respectivement les valeurs des bilans hivernaux et bilans annuels, en mètres d'eau.

\section{IV — LE MASSIF DES GRANDES ROUSSES}

Dans ce massif, deux glaciers font l'objet de suivis à long terme : il s'agit des glaciers de Sarennes et de Saint Sorlin.

Celui de Sarennes est suivi pour son bilan de masse depuis 1949 par le Cemagref [11] et celui de Saint Sorlin pour son bilan et sa dynamique depuis 1956 par le Laboratoire de Glaciologie [10].

On dispose ainsi sur ce massif des relevés en deux glaciers différents en taille $\left(0,5\right.$ et $\left.3 \mathrm{~km}^{2}\right)$ et en exposition (Sud et Nord).
6. Glacier de Gébroulaz : Variations des Altitudes moyennes des 3 profils E\&F de 1907 à 1999.

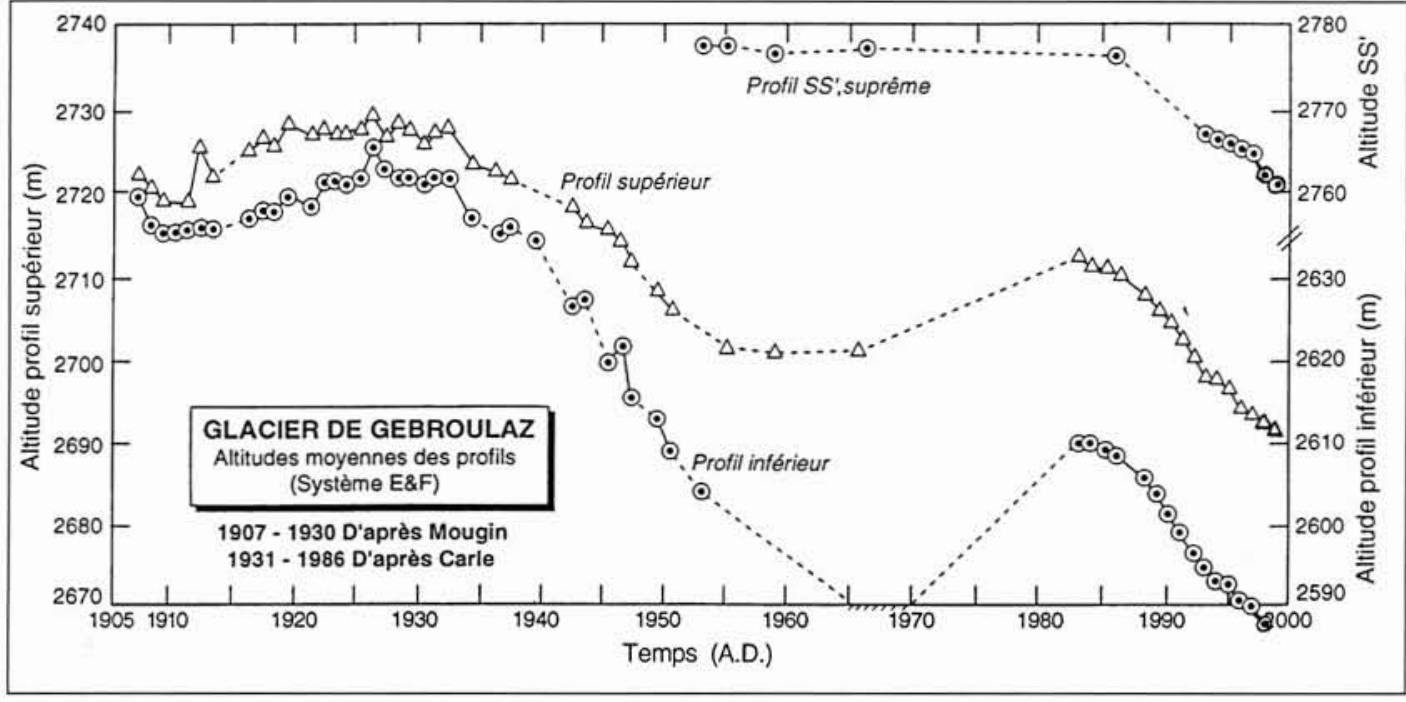


7. Glacier de Gébroulaz : variations des vitesses moyennes annuelles sur les 3 profils $E \& F$ de 1908 à 1999 .

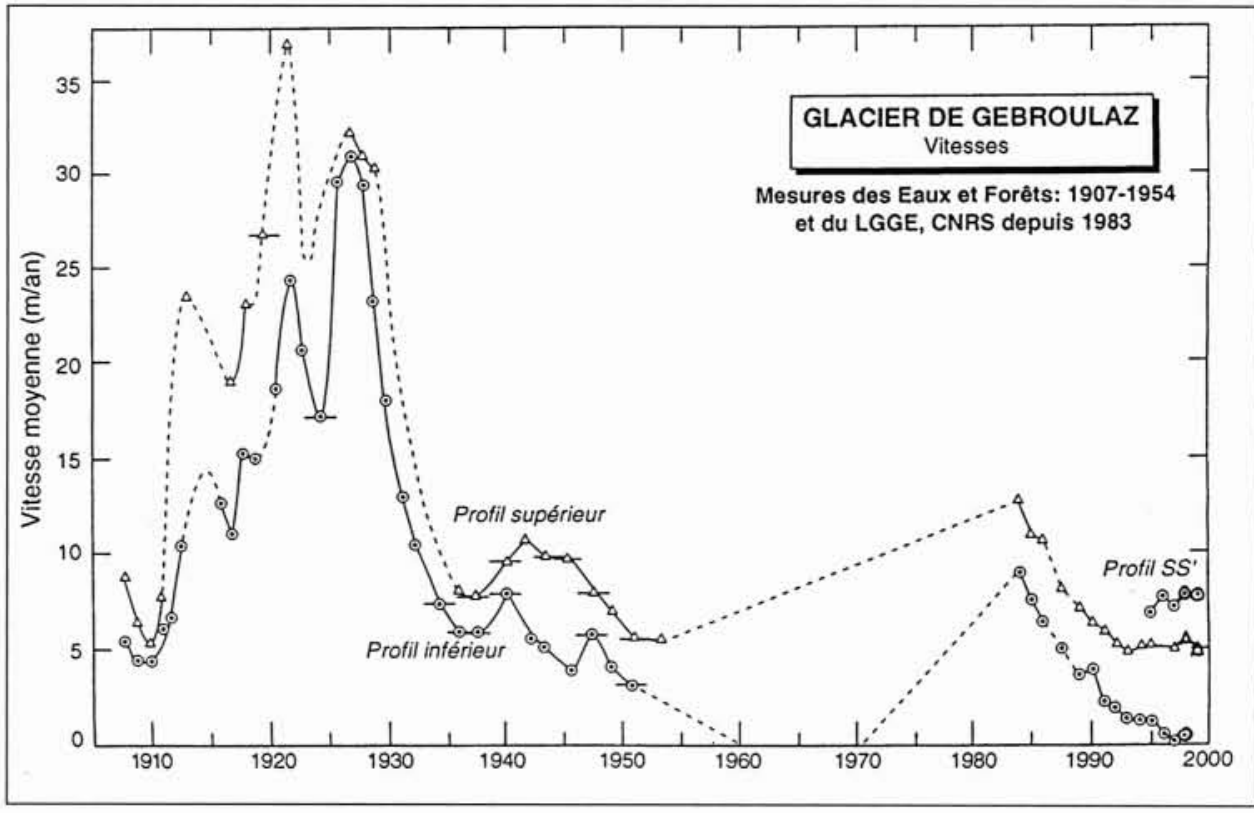

Effectivement leurs bilans annuels sont différents : sur la période commune de comparaison de 1957 à 1999 (42 ans), les bilans moyens cumulés, portés sur la fig. $\mathrm{n}^{\circ} 8 \mathrm{a}$, montrent une perte pour Sarennes de 25 mètres d'eau $(60 \mathrm{~cm} / \mathrm{an})$, et de 15,5 mètres d'eau $(37 \mathrm{~cm} / \mathrm{an})$ pour Saint Sorlin.

Toutefois on note une forte ressemblance dans le déroulement des changements de bilan, avec des instants de variations communs caractéristiques, comme en 1965,77 et 95 .

En utilisant le modèle linéaire de comparaison des fluctuations des bilans de masse proposé par Lliboutry en 1974 [12] et en l'étendant à la comparaison de différents glaciers, on peut comparer les écarts à leur propre moyenne de ces deux appareils (bilans centrés).

Ainsi sur la figure $n^{\circ} 8 b$, les cumuls des bilans centrés donnent une évolution très semblable pour ces deux glaciers voisins : il semble donc que, malgré les différences de morphologie des glaciers, de méthode de suivi, on mesure bien les mêmes effets climatiques annuels.

Cette caractéristique de la distribution spatio-temporelle des bilans de masse glaciaire est en fait beaucoup plus étendue que le massif des Grandes Rousses : elle se retrouve d'un bout à l'autre du massif alpin [13, 14]).

\section{V $\square$ MASSIF DES ECRINS}

Dans le massif des Ecrins, les relevés du type E\&F portaient sur les Glaciers Blanc, Noir, Pilatte, Chardon et Selle. Le LGGE avait repris en 1983 les mesures E\&F sur les glaciers Blanc et Noir, mais du fait d'un recul important au front du glacier Blanc et à l'atterrissement de son profil inférieur, seul le glacier Noir continue à mériter un suivi à 2 profils.

En revanche le glacier d'Arsine bénéficie de relevés depuis les années 1986 tant pour le glacier (bilan de masse, altitudes et vitesses) que pour les variations du lac proglaciaire [15]. Ce suivi fait maintenant l'objet d'une attention plus particulière dans le cadre de l'étude des risques naturels d'origine glaciaire.

Les variations de longueur récentes du glacier Blanc, portées sur la figure $n^{\circ} 9$, de 1950 à 1999 , illustrent bien la

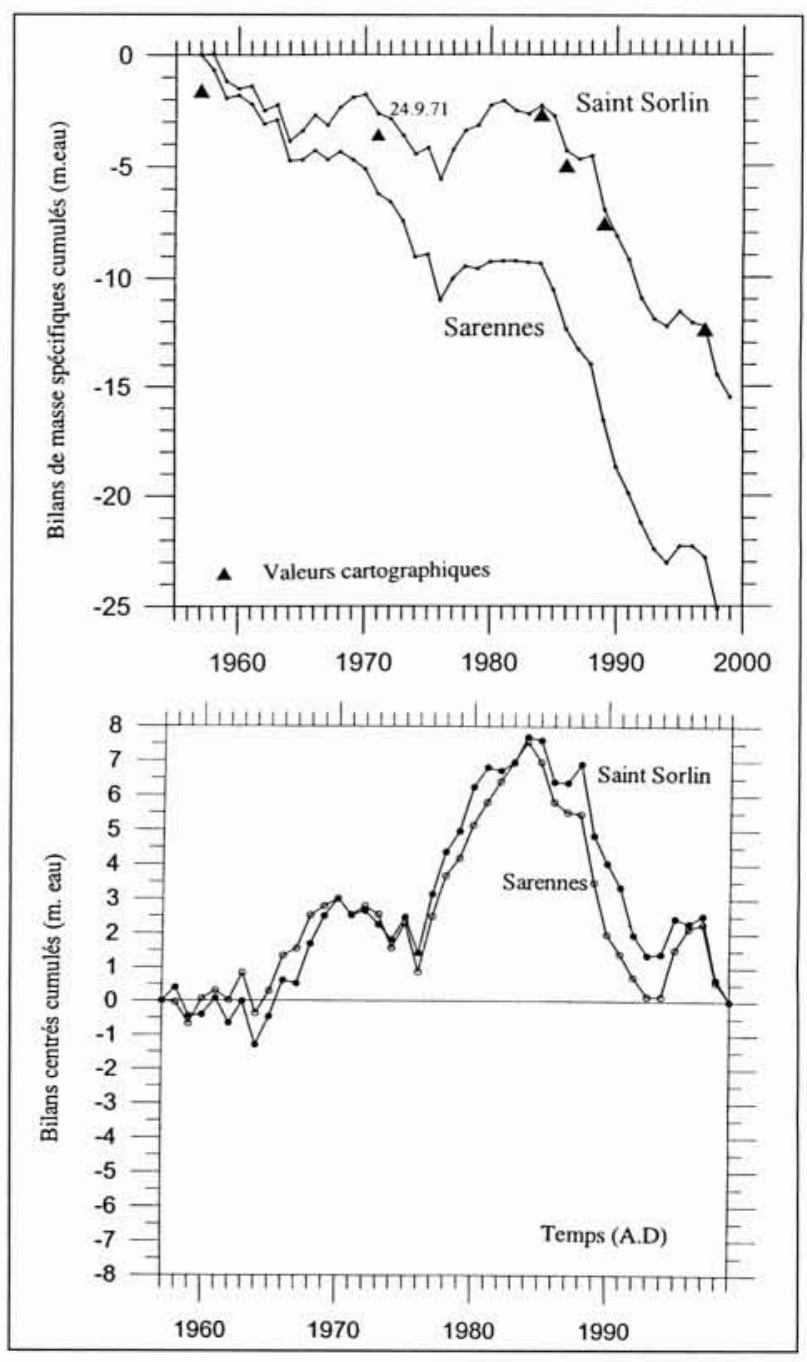

8. Comparaison des bilans de masse relevés sur les glaciers de Sarennes (ETNA-Cemagref) et de Saint Sorlin (LGGE-Cnrs) :

a - évolution des bilans cumulés : 1957 à 1999 ,

b - variation des bilans centrés cumulés sur la même période. 


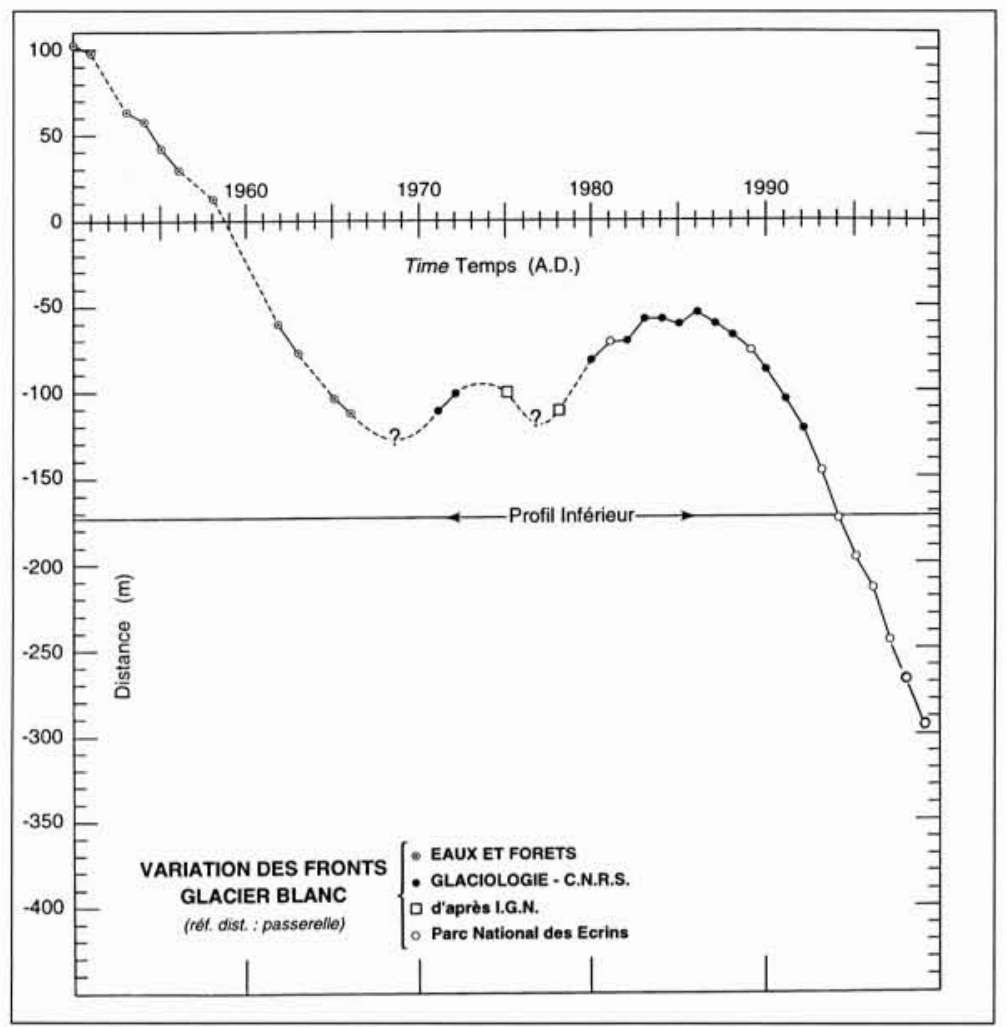

9. Glacier Blanc :Variations de longueur récentes depuis $\mathbf{1 9 5 0 .}$ Mesures des E\&F, du LGGE-Cnrs et du Parc National des Ecrins.

reprise d'avancée des années 80 et le fort recul actuel (disparition du profil inférieur en 1994).

Parmi les autres longues séries de variations glaciaires de ce massif, on compte celle du glacier de la Pilatte, portée sur la figure $n^{\circ} 10$, des années 1840 à 1999 , avec la série des mesures récentes effectuées par le Parc National des Ecrins.

\section{VI $\square$ COMPARAISON DES FLUC- TUATIONS DE LONGUEUR LE LONG DES ALPES FRANÇAISES}

Sur la figure $\mathrm{n}^{\circ} 11$ sont portées les fluctuations de longueur de 3 glaciers situés respectivement dans le massif du Mont-Blanc (Argentière), le massif de la Vanoise (Gébroulaz) et le massif des Ecrins (Blanc), pour la période où les glaciers ont commencé à être suivis assez régulièrement (\# 1870).

Ces trois glaciers montrent des fluctuations assez semblables, où l'on retrouve les caractéristiques principales des réavancées de 1890,1920 et 1980, puis la grande décrue des années 40 à 50 .

Il semble donc, que pour des glaciers d'une taille comparable à celle de ces trois appareils. on retrouve tout le long des Alpes les mêmes conséquences de variations climatiques homogènes sur tout le massif Alpin comme le montre l'analyse de distribution des bilans de masses glaciaires.

\section{VII — CONCLUSIONS}

Dans les Alpes Françaises, les suivis de variations glaciaires portent sur un nombre assez restreint de glaciers répartis dans les quatre principaux massifs, depuis le Mont-Blanc jusqu'aux Ecrins : actuellement, les bilans de masse annuels sont mesurés sur 6 glaciers (Argentière, Mer de Glace, Gébroulaz, Saint Sorlin, Sarennes (ETNA-Cemagref) et Arsine). Cinq glaciers (les mêmes sauf Sarennes) font l'objet de mesures de vitesses et de variations d'épaisseurs. Enfin les variations de longueur sont effectuées sur 10 glaciers.

Grâce aux mesures déjà effectuées depuis les années 1900 (longueurs, altitudes et vitesses), principalement par l'administration des E\&F, aux mesures de bilan de masse plus récentes depuis les années 1949 pour Sarennes, 1956 pour Saint Sorlin et aux possibilités offertes par les suivis complets en cours sur les grands glaciers du Mont-Blanc, couvrant une large gamme d'altitudes allant de $1500 \mathrm{~m}$ à plus de $3000 \mathrm{~m}$, on dispose aujourd'hui d'un ensemble de données très significatif pour décrire les réactions des glaciers aux changements du climat.

Ces séries d'observations révèlent quelquefois des comportements assez inattendus de la dynamique des glaciers comme les accélérations du glacier de Gébroulaz au cours des années 20 et d'autre part permettent d'avoir un panorama complet des fluctuations des glaciers au cours du temps. Cela constitue une vérité terrain indispensable dont aucun modèle théorique n'est encore capable de rendre compte [10].

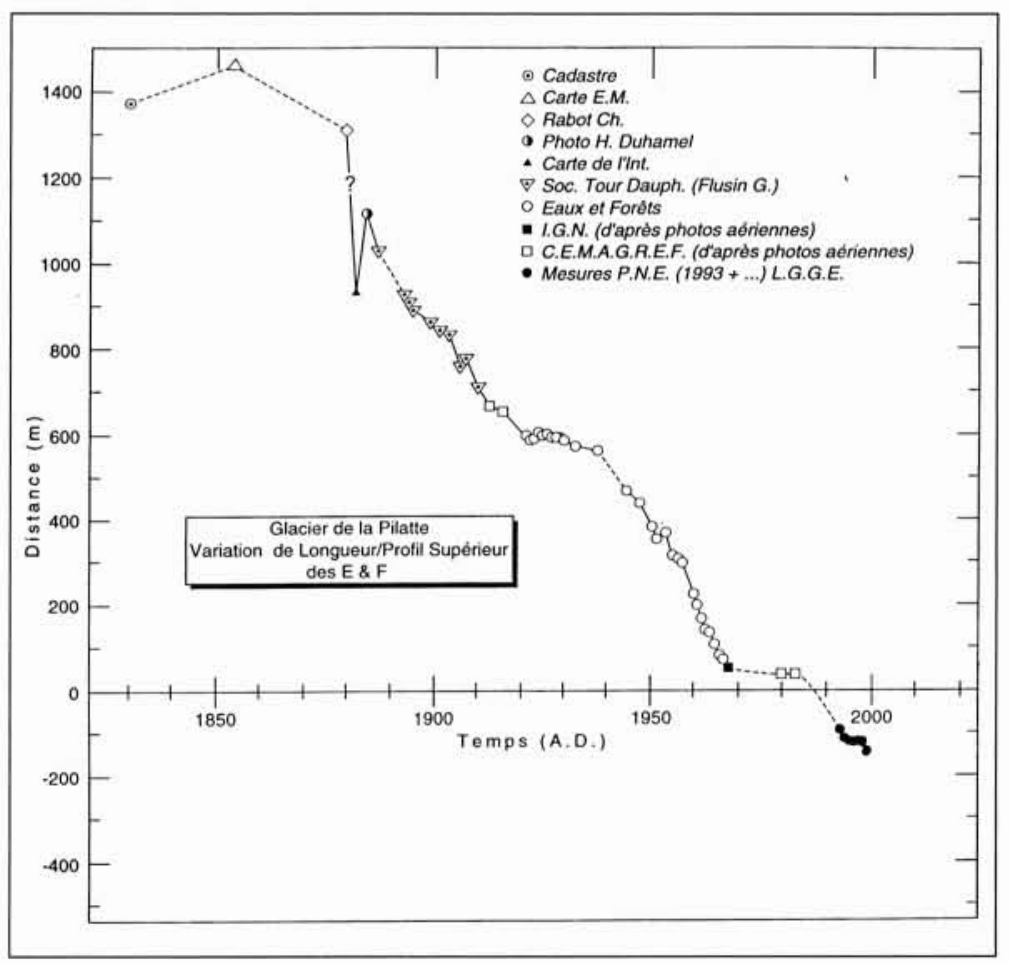

10. Variations de longueur du Glacier de la Pilatte, suivi récent effectué par le Parc National des Ecrins. 


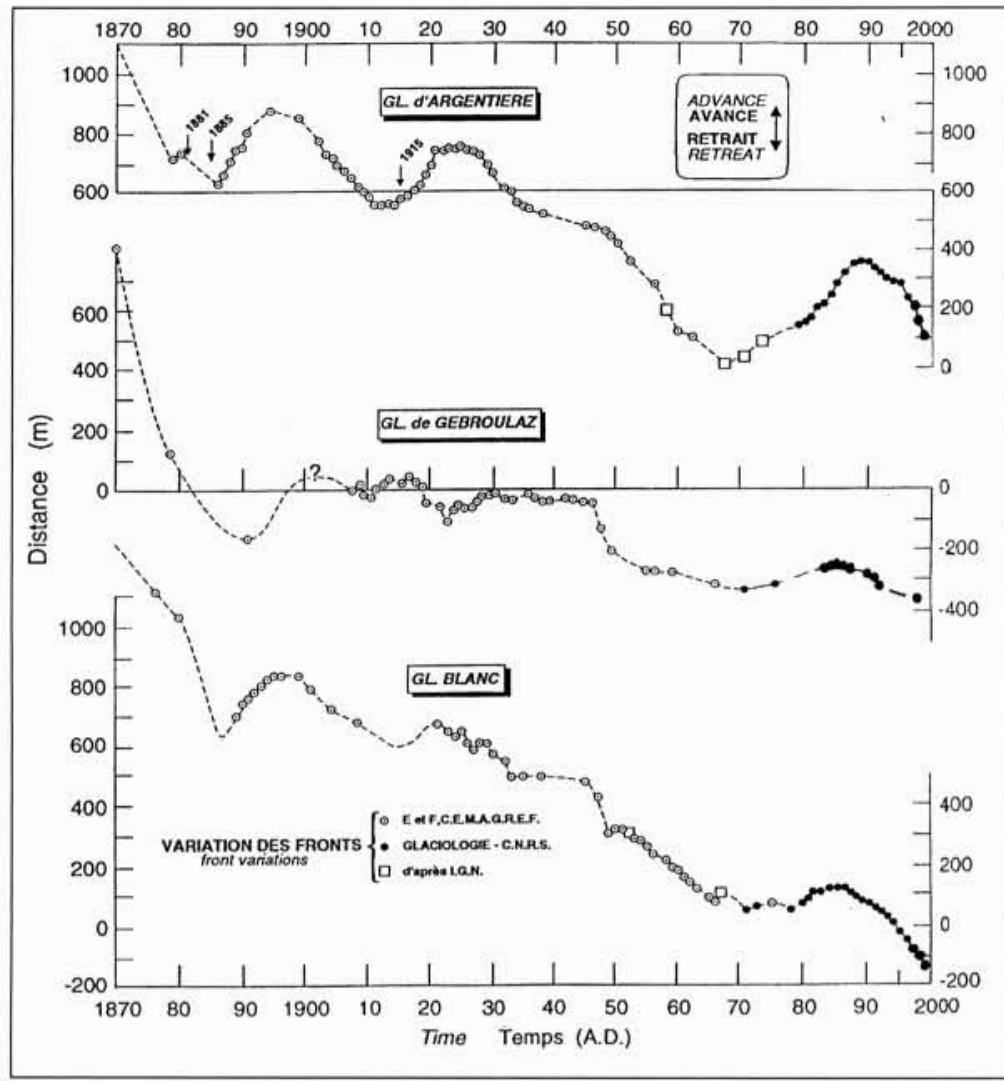

11. Variations de longueur comparées sur le dernier siècle de trois glaciers des Alpes Françaises : Argentière (Mont-Blanc), Gébroulaz (Vanoise) et Glacier Blanc (Ecrins).
C'est pourquoi il nous apparaît absolument nécessaire de compléter cette base de données en continuant un suivi assidu de cet échantillon de glaciers, assez représentatifs de la diversité des appareils ainsi que de la variété des situations géographiques et climatiques.

Ces données de base sont bien sûr nécessaires pour la recherche fondamentale sur les mécanismes de base qui régissent les fluctuations glaciaires, valables autant pour les glaciers alpins que pour les grandes calottes glaciaires. De plus, elles trouvent des applications pratiques importantes dans la résolution des problèmes posés par l'étude des risques naturels glaciaires ou encore de ceux rencontrés dans l'aménagement moderne de la haute montagne glaciaire.

\section{Remerciements}

Ces ensembles de données n'ont pu être constitués que grâce à la persévérance au cours du temps d'organismes comme les E\&F, le Cemagref et le CNRS, ainsi qu'à l'intérêt des industriels (Emosson SA, EDF, Grands Montets. Montenvers) et à l'attention des Parcs Nationaux de Vanoise, des Ecrins et à celle des Réserves Naturelles de Haute Savoie.

Enfin la collecte des innombrables relevés annuels n'a pu se réaliser que grâce à la participation d'un grand nombre de personnes, à titre d'expert ou de bénévole, qu'il faut remercier chaleureusement.

\section{RÉFÉRENCES}

[1] Mougin P., 1910, Etudes glaciologiques, Tome 1, Paris, Direction Générale des Eaux et Forêts, Service des forces Hydrauliques, Imprimeric Nationale.

[2] Reynaud L., 1985, Le Glacier de Tré-la Tête, Editions du Comité Scientifique des Réserves Naturelles de Haute Savoie, brochure, 16 p.

[3] Vallon M., Vincent C. et Reynaud L., 1995, Sensibilité des bilans de masse glaciaires aux fluctuations climatiques. La Houille Blanche. $n^{\circ} 5 / 6$ 1995, p.167-174.

[4] VAllon , M., VINCENT C. and REynaud L.,1998, Altitudinal gradient of mass-balance sensitivity to climatic change from 18 years of observations on Argentière glacier (France). Journal of Glaciology, vol 44, $\mathrm{n}^{\circ}$ 146. p. $185-190$

[5] VALLOT J., 1900, Expériences sur la marche et la variation de la Mer de Glace.. Annales de l'Observatoire Météorologique, Physique et Glaciaire du Mont-Blanc, Vol. 4, pp.1815-1817

[6] Lliboutry L. and Reynaud L., 1980, Global dynamics of a temperate valley glacier, Mer de Glace and past velocities using Forbes bands. Journal of Glaciologie, 27, 96, p. 207-226.

[7] ReYNAUd L., 1980, Reconstruction of past velocities using Forbes Bands (Mer de Glace), Zeitschrift für Gletscherkunde und Glazialgeologie, Band 15, Helft 2, p. 207-226.

[8] Tournoud M., 2000, Reconstitution des vitesses dans le passé à l'aide de la disposition des Bandes de Forbes sur la Mer de Glace (France) et le Glacier de l'Unteraar (Suisse), Réunion de la SHF-Glaciologie-Nivologie, mars 2000, Grenoble, non publié
[9] ReYnaud L., Vincent C. et Vallon M., 1998, Mesures récentes effectuées sur le glacier de Gébroulaz (massif de la Vanoise. France), depuis 1978, par le Laboratoire de Glaciologie du CNRS concernamt les variations d'altitude, de vitesse, de bilan de masse et la mise en place d'un réseau de repères topographiques pour l'utilisation de la photogrammé. trie aérienne. Travaux Scientifiques du Parc National de la Vanoise. Tome $\mathrm{n}^{\circ} \mathrm{XX}, \mathrm{pp} .83-109$

[10] Vincent C., Vallon M. et Reynaud L.. 2000, Dynamic behaviour of the glacier de Saint Sorlin (France) from 40 years of observations. 1957-1997, In press : Journal of Glaciology.

[11] VALLA F., 1995. The mass balance of glacier de Sarennes, Zeitschrift fur gletscherkunde und glazialgeologie, band 31 (1995) S.189-197

[12] LLiboutRy L., 1974, Multivariate statistical analysis of glacier annual balances, Journal of Glaciology, Vol, 13, n69, pp. 371-92.

[13] Letregull.ty A. and Reynaud L.. 1990, Glaciers mass balance in the northern hemisphere: space and time distribution. Arctic and Alpine Research, Vol. 22, n 1 , p. 43-50

[14] VINCENT C.. 1995, 38 ans de bilans de masse glaciaire (1956-1994) sur 2 glaciers du massif des Grandes Rousses., Società Meteorologica Subalpina, Nimbus, $n^{\circ} 8, p .15-18$.

[15] VALLoN M., 1989. Evolution, water balance, potential hazards and control of a proglacial lake in the French Alps (Arsine). Journal of Glaciology, 1989, 13, 273-278.

16] Reynaud L.. Vallon M. and Letréguilly A.. 1987, Mass balance measurements : problems and two new methods of determining variations, Journal of Glaciology, vol, 32, $\mathrm{n}^{\circ}$ 112, p. 446-454 